\title{
PERSPECTIVAS SOBRE WEIMAR
}

\section{PERSPECTIVES ON WEIMAR}

\author{
Ignacio Gutiérrez Gutiérrez \\ UNED (Universidad Nacional de Educación a Distancia)
}

Recensión de / Review of: Jesús Casquete y Javier Tajadura (coords.), La Constitución de Weimar: Historia, politica y derecho, CEPC, Madrid, 2020, 291 pp.

Palabras clave: Weimar, Constitución de Weimar, República de Weimar

Key Words: Weimar, Weimar Constitution, Weimar Republic

I. Weimar no es solo una ciudad. Es también un simbolo, como ocurre (en distinta medida y por razones muy diversas) con muchas otras ciudades. Lo era ya cuando se decidió que fuera el lugar de reunión de la Asamblea Nacional constituyente elegida en Alemania el 19 de enero de 1919. En esa decisión estaba implícita la voluntad de aprovechar tal valor simbólico en beneficio de la nueva República: la Asamblea reunida en Weimar produciría la Constitución de Weimar, que regiría los destinos de la República de Weimar.

Ocurre, sin embargo, que el uso de un simbolo afecta a su significado, depositando sobre él nuevas referencias que no siempre lo confirman, lo modulan o lo complementan; a veces también pueden degradarlo, sustituirlo e incluso invertirlo. Los historiadores analizarán luego los estratos de sentido acumulados en la palabra; pero esta nunca volverá a recuperar su valor originario, el símbolo siempre arrastrará consigo toda su trayectoria. Cada nuevo uso habrá de contar con ella y, en su caso, explicitar la perspectiva elegida. ¿Es Weimar la ciudad de Goethe y Schiller? ¿La de la Asamblea constituyente y la naciente República? ¿O la de su fracaso final, la del cercano Buchenwald?

También el texto constitucional adoptado en Weimar se convirtió enseguida, como es propio de las constituciones, en un simbolo, sobre el que se proyectaban las experiencias y las expectativas colectivas, pero también las frustraciones y los fracasos del pasado inmediato. Sobre él se fueron acumulando luego sucesivos estratos de sentido, a medida que la norma constitucional iba cobrando significados concretos en su contraste con las realidades políticas y sociales del momento. Y finalmente, agotada su vigencia, la Constitución de Weimar siguió siendo una referencia simbólica; no solo para el régimen nacionalsocialista que la desplazó, sino también, y de modo particular, para la nueva República Federal que se puso en marcha bajo el control de las potencias aliadas occidentales: Bonn no debía ser Weimar. Aún hoy, la Constitución de Weimar sigue proyectando sus luces y sus sombras: Weimars lange Schatten es el título de un libro colectivo editado por Christoph Gusy en 2003 (Baden-Baden, Nomos), del que esta misma Revista, en su número 9, extrajo para su publicación una significativa contribución de Oliver Lepsius: "El redescubrimiento de Weimar por parte de la doctrina del Derecho político de la República Federal" (DOI: https://doi.org/10.17811/hc.v0i9.152). 
Las conmemoraciones del centenario de su aprobación, cumplido en el verano de 2019, han multiplicado la bibliografia sobre la Constitución de Weimar. Baste aquí con mencionar, como muestra, el dossier coordinado por Leonardo Álvarez en el n. ${ }^{\circ} 20$ de esta Revista (DOI: https://doi.org/10.17811/hc.v0i20). Y, cada vez que se vuelve la vista hacia ella, su significado va reconfigurándose conforme a la perspectiva que transmite el nuevo observador. Ningún libro consagrado al tema puede escapar de esa cadena de creación de sentido; porque incluso el investigador más empeñado en el contraste exhaustivo de las fuentes y en su transmisión rigurosa desarrolla también una tarea de selección, sistematización e interpretación; con ella se incorpora al discurso colectivo que constituye nuestra única vía de acceso al pasado. La recensión de una obra sobre Weimar tiene así un objetivo inexcusable: identificar las capas de significado que sus autores pretenden colocar en primer plano y el modo en que, con ello, contribuyen a la reconstrucción del pasado y a su percepción actual.

II. El título de este libro dirige la atención precisamente hacia "la Constitución de Weimar". Pero el objeto de estudio se concibe en términos muy amplios; pues, de acuerdo con lo que afirman los coordinadores en la "Presentación", se trata de acercarse "a la génesis, fundamento, contexto y contenido de dicho texto constitucional". También el enfoque se diversifica, porque se busca "una aproximación interdisciplinar desde el Derecho, la Historia y la Ciencia Política". El subtítulo del libro, "Historia, política, derecho", identifica así métodos distintos con objetos también diferentes; unidos, eso sí, por un punto común de referencia: el texto constitucional de Weimar.

En esa perspectiva, la obra se divide en tres partes. La primera comprende casi la mitad de su extensión y se compone de un único capítulo, "La República de Weimar: Constitución y contexto" (pp. 17-138), que firman conjuntamente los dos coordinadores del libro, ambos adscritos a la Universidad del País Vasco, uno historiador y otro jurista (Jesús Casquete es profesor de Historia del pensamiento y de los movimientos sociales y politicos y Javier Tajadura profesor de Derecho constitucional. En él abordan, en sus propias palabras, el "contexto histórico, político y social", "el proceso de elaboración de la Constitución y las líneas maestras de la carta magna", y también su desenvolvimiento "durante los primeros años de andadura del nuevo régimen". Su pretensión es "hacer comprensible a un público hispano el contexto en el que discurrieron el debate y la aprobación de la Carta Magna, así como las líneas maestras de su contenido", centrándose "en la primera fase de la República", sin considerarla "a partir de su final".

Pero lo cierto es que, a la hora de seleccionar, presentar y valorar los hechos pasados, no resulta fácil situarse en el contexto de la época y desprenderse del conocimiento, solo después accesible, acerca de la evolución posterior de los acontecimientos. Lo reconocen los autores en los primeros párrafos de su contribución: "no es posible disociar ambas dimensiones". Y el capítulo termina precisamente con un "Epílogo: el colapso de la república" (pp. 126-134), que documenta la crisis económica y social, politica y, con mayor detalle, de orden público (enlazando aquí con el texto posterior de Ángel Alcalde) que dominó el panorama desde 1929. Ocurre así que, evitando mirar Weimar desde 1933, se termina adoptando la misma perspectiva de quienes asumieron, en 1949, la labor de reconstruir Alemania occidental y darle una nueva Ley Fundamental. Y por eso se dice que, aunque "las causas del fracaso de la Constitución de Weimar no 
deben buscarse en su texto", "su recuerdo, memoria y enseñanza han sobrevolado la historia del país después de la pesadilla nacional-socialista. Weimar constituye el modelo de lo que no puede volver a repetirse".

La descripción del contexto histórico en el que se adopta la Constitución resulta, en cualquier caso, instructiva. El capítulo esboza el modelo institucional diseñado por la Constitución de 1871 y el intento de reorientarlo a la vista de la situación bélica que se constataba ya en octubre de 1918: demasiado tarde. Relata las insubordinaciones y revueltas que comenzaron en Kiel y se extendieron por toda Alemania, los acontecimientos politicos de Berlín que culminaron con el traspaso del poder a Friedrich Ebert y la proclamación de la República por Philipp Scheidemann y por Karl Liebknechk (de forma no solo separada, sino también contradictoria), la formación de los consejos y su papel en la gestión de la quiebra y la derrota militar, la convocatoria de la Asamblea Nacional constituyente y la disidencia espartaquista que desembocó en el levantamiento de enero de 1919 en Berlin. También resume la normativa que rigió las elecciones del 19 de enero y su resultado; pero en este contexto expone, con mucho mayor detalle (pp. 32-44), el panorama de las fuerzas políticas que se enfrentaron en ellas (antecedentes y continuidades, bases sociales y posiciones fundamentales, evolución posterior, alianzas de gobierno, etc.) e incluso de algunas surgidas más tarde (partido nacionalsocialista), y también estudia el voto femenino que por primera vez se reconoce en esas elecciones (incluyendo en el análisis todo el periodo de la República). Se trata de un largo inciso que enlaza con el posterior capitulo de Jürgen W. Falter.

El texto continúa refiriendo la elección de Weimar como lugar de reuniones, la apertura de las sesiones el 6 de febrero, la aprobación de la ley de poderes provisionales del Reich, en la que la Asamblea se autoatribuia el poder constituyente. Sobre ella se puso en marcha un nuevo orden institucional que en cierto modo representa la continuidad entre la previa organización del Imperio y la que luego instaurará la Constitución: poder legislativo compartido por la Asamblea con una Comisión de Estados, Presidente del Reich (Ebert) y gabinete ministerial (Scheidemann, luego Bauer). El capítulo se refiere igualmente a los graves desafios que tuvieron que afrontar esos poderes provisionales: económicos, sociales y de orden público, pero también, y decisivamente, tomar posición frente al Tratado de Versalles, de cuyo simbolismo y contenido se da también cuenta detallada. El Tratado "proyectó su tenebrosa sombra sobre la República", y la Constitución, vinculada al mismo, "se identificó desde su inicio con la humillación y la derrota".

Se documenta, por supuesto, el proceso que condujo a la aprobación del texto constitucional: los grupos politicos implicados en las negociaciones (que enseguida perdieron su posición de dominio y no lograron convertirse así "en el fundamento social y politico que garantizase la estabilidad de la república"), los trabajos preliminares de Hugo Preu $\beta$ y sus posiciones fundamentales, los acuerdos con los Estados federados y las sesiones de la Asamblea dedicadas a debatir el proyecto (a las que también podian asistir los miembros de la Comisión de Estados), la aprobación final del texto (26 de julio) y la firma del Presidente en su lugar de vacaciones (11 de agosto). Y, de inmediato, las dificultades, comenzando por la crisis gubernamental desencadenada por la firma del Tratado de Versalles, antes por tanto de la adopción del texto constitucional, y siguiendo por las agitaciones politicas y sociales de los años inmediatos, entre los golpes de estado de Kapp (1920) y de Hitler (1923), marcados por los levantamientos obreros y el terrorismo contrarrevolucionario. 
También se analiza con detalle el contenido de la Constitución, desglosado en cinco grandes apartados: la forma de gobierno democrática-parlamentaria y los instrumentos de democracia directa (pp. 76-82), la posición constitucional del Presidente del Reich (pp. 83-95), el orden federal (pp. 96-109), la reforma constitucional y el control de constitucionalidad de las leyes (pp. 110-119) y, finalmente, los derechos fundamentales, con atención particular al modo en que se abordan el estatuto de las confesiones religiosas y la educación (pp. 119-126). En cada uno de esos temas se recogen las posiciones de partida de los grupos políticos participantes en el proceso constituyente, se detalla la regulación contenida en el texto, se exponen los problemas que tal normativa deja planteados y se explica asimismo el modo en que fueron abordados y resueltos en la práctica; pero no solo en el periodo inmediato a la aprobación de la Constitución, como anunciaban los autores, sino a lo largo de toda su vigencia. De este modo, la perspectiva del momento final se impone sobre toda la exposición. Y así se vincula, por ejemplo, la explicación del clima intelectual en el que se constitucionaliza la institución presidencial de legitimación plebiscitaria con la deriva presidencialista que caracteriza el periodo final de la República, de la que se responsabiliza de modo unilateral al sistema electoral proporcional y al comportamiento de los partidos políticos. Y, del mismo modo, los poderes excepcionales atribuidos al Presidente por el art. 48 de la Constitución (pp. 89-95 y 102-109, donde se abordan temas que más adelante tratan con detalle Leonardo Álvarez y Leticia Vita) se valoran fundamentalmente desde la perspectiva de su manipulación por parte de los gobiernos no parlamentarios de los últimos años.

En definitiva: aunque se dice: "la república navegó sin rumbo. Y, a falta de rumbo, encalló", el juicio contenido en la primera proposición viene determinado, precisamente, por el conocimiento que nos proporciona, a posteriori, la segunda. El acercamiento al contenido de la Constitución de Weimar parte de su desenlace y de nuestra experiencia, y ello pese a la pretensión de alejarse de tal perspectiva.

En su planteamiento explícito, tal pretensión podría formalizarse, siguiendo a Konrad Hesse y a Pedro Cruz, como un análisis de las condiciones iniciales de realización de la Constitución y una mirada a su primer periodo de vigencia, en el que habría de cobrar su verdadera y peculiar cualidad constitucional. Es un acercamiento que ha mostrado su productividad a la hora de explicar el significado y el alcance de las constituciones normativas del Estado social y democrático de Derecho; y aquí se pretende proyectar sobre lo que los autores consideran precisamente como "el texto fundacional del constitucionalismo democrático y social", "faro" y "guía para la elaboración de las constituciones aprobadas en Europa al término de la II Guerra Mundial". Pero lo cierto es que esa misma calificación supone una anticipación, de la que naturalmente no podía haber consciencia en la época. El primer epígrafe de este capítulo se dedica precisamente a caracterizar ese "nuevo tipo histórico de constitución: la constitución democrática"; y ese modelo inspira el juicio que merece luego el texto constitucional recién aprobado, cuyo valor se identifica con su "carácter de compromiso" y sus "decisiones a favor de la libertad y de la democracia". Pero, al contemplar Weimar desde el paradigma de la Constitución rígida y dotada de supremacia, garante de los derechos fundamentales y del pluralismo, integradora del conflicto social y desprendida del mito de la soberanía, se están prejuzgando, 
con la mirada de nuestros días, los debates teóricos y los conflictos reales en los que cobró significado el texto de 1919.

Si el texto se contempla de ese modo, es natural concluir que sencillamente no se correspondía "con la situación política y social", encontraba el rechazo de "numerosos sectores políticos y sociales" y resultaba por eso "inadecuado para resolver los graves conflictos que dividian a la sociedad alemana". El capítulo concluye afirmando que los poderes reales (funcionariado, judicatura, fuerzas armadas, sistema educativo) operaron como una constitución real (en el sentido de Lasalle) que se impuso al texto escrito: "nostálgicos del antiguo régimen, y sin convicciones democráticas, acabaron abrazando el nazismo". La Constitución no pudo garantizar el compromiso, ante "la ausencia de las condiciones" necesarias para ponerla en práctica. No es casual que se invoque en este punto el juicio ofrecido precisamente en 1946 por Costantino Mortati, el célebre autor de La costituzione in senso materiale (1940).

Una atención más diferenciada a los debates doctrinales de la época quizá pudiera haber generado un acercamiento menos idealista al texto constitucional. Se hace evidente, en particular, con la insistencia de los autores en el valor supralegal de las Constituciones democráticas, que resultó discutido y cuando menos precario en el caso de la de Weimar (se analiza en pp. 112-119), como lo eran las concepciones de la democracia pluralista y de la Constitución como compromiso que se identifican como caracterizadoras del modelo. También podrian cobrar otro sentido, en ese contexto, los análisis sobre el sesgo conservador de la judicatura y los particularmente detallados (pp. 65-76) sobre las debilidades simbólicas de la nueva República, cuya trascendencia se subraya y que se reflejaron en las sostenidas disputas acerca de la bandera y en las tibias celebraciones anuales del 11 de agosto. No resultó aquí suficiente que la Asamblea Nacional hubiera optado por mantener en la rúbrica de la Constitución la denominación de Alemania como Reich.

III. Las otras dos partes de la obra contienen cinco breves contribuciones sobre el "contexto social y político" y sobre "cuestiones constitucionales controvertidas". Diferentes especialistas abordan aquí problemas muy particulares: fluctuaciones electorales, paramilitarismo y antisemitismo en el primer bloque, poderes excepcionales del Presidente del Reich y proceso judicial sobre la intervención federal en Prusia (Preußenschlag de 20 de julio de 1932) en el segundo. Los coordinadores son conscientes de que este elenco de contribuciones no puede "hacer justicia a la complejidad del periodo", pero consideran que los temas son "oportunos para acceder a una cabal comprensión de la época y de su final". Las palabras con las que Leticia Vita da comienzo a su texto, el último del volumen, insisten en el sentido que los coordinadores atribuyen al conjunto de esas cinco contribuciones: "La historia de la República de Weimar cuenta con muchos capitulos. Sin embargo, tal vez a sabiendas de su trágico final, nos ocupamos especialmente de aquellos que precipitaron su caída". Así, la mirada histórica, que la primera parte del libro pretendía situar en la fase inicial de la República de Weimar, cuando el futuro era incierto, se desplaza explícitamente aquí hacia sus últimos momentos, cuando nuestro conocimiento del desenlace, inaccesible para quienes convivian con los acontecimientos del periodo, incita a centrar la atención en los hilos que condujeron a la catástrofe. Aquí ya no pretende ofrecerse, pues, un panorama representativo de la vida constitucional (sociedad, politica, derecho) a lo largo de la República de Weimar; 
sino que la selección recae precisamente sobre tramas cuya trascendencia se acreditó solo al final del relato.

El texto de Jürgen W. Falter, catedrático de Ciencia Política en Mainz y especialista en sociología electoral, lleva por título "La base social de los ejes de conflicto en la República de Weimar, 1919-1933" (pp. 141-167). Comienza con una serie de consideraciones conceptuales acerca del significado atribuido a las expresiones "subcultura politica" (Lehnert y Megerle), "campo político" (Burnham), "ejes de conflicto" (Pappi) y "entorno socio-moral" (Lepsius); pero el núcleo de su exposición consiste en valorar, mediante el análisis cuantitativo de los resultados electorales y de las transferencias de votos, el peso relativo de las diferentes subculturas y sus fluctuaciones.

El autor constata, de un lado, una considerable estabilidad de los bloques electorales durante la vida de la República, incluso con respecto al periodo anterior (con la salvedad precisamente de las elecciones de 1919 de las que surgió la Asamblea constituyente) y hasta 1932; de otro, la relativa transversalidad (con la salvedad aquí del voto católico) de las aportaciones al crecimiento del partido nacionalsocialista, situado en todo caso en el entorno del bloque burgués-protestante. También analiza la composición de los respectivos electorados atendiendo a la clase social (agrario, cuello azul, cuello blanco y autónomo) y a la confesión religiosa (protestantes y católicos). Desde este punto de vista, el voto católico está notablemente agrupado; pero la clase social tiene una incidencia matizada: los votos de los partidos de izquierda tienen un origen más limitado, fundamentalmente procedente de grupos (cuello azul para el partido comunista, azul y también blanco en el caso del partido socialista) que, sin embargo, no concentran el voto en tales partidos, sino que lo distribuyen entre todos los demás. También en este sentido se constata, pues, la heterogeneidad de los votantes del partido nacionalsocialista.

Ángel Alcalde, profesor de Historia contemporánea en la Universidad de Melbourne, analiza con detalle "El paramilitarismo en Weimar" (pp. 169-196). Tras una aproximación conceptual y contextual breve, clara e iluminadora, va desgranando las distintas fases por las que atraviesa el fenómeno, que dan lugar a distintos tipos de organización paramilitar y que también tienen diferentes efectos sobre el devenir histórico.

Aunque el autor destaca también la disponibilidad de medios (personales y armamentísticos) como condición de posibilidad para el surgimiento del paramilitarismo, sus origenes vienen determinados por el papel que desempeñaron los Freikorps en el inicial asentamiento de la República. Su instrumentalización por parte de las nuevas autoridades sirvió para reprimir los desafios radicales (Berlín, Múnich). Pero esa legitimación institucional de la violencia paramilitar, con un valor simbólico dificilmente reversible, en absoluto permitió a la República ganarse la lealtad del estamento militar y de los grupos armados vinculados a él, aferrados a la convicción de haber sido traicionados por la retaguardia al final de la guerra, ofendidos por el Tratado de Versalles y huérfanos ya de la referencia que suponian el Kaiser y la bandera, como puso de manifiesto de inmediato el golpe de Estado de marzo de 1920 (Kapp-Putsch).

$\mathrm{Su}$ fracaso abrió un paréntesis solo aparente, en el que proliferaron los grupos paramilitares y, siguiendo el modelo del partido fascista italiano, se formaron las SA (Sturmabteilung o sección de asalto del partido nacionalsocialista), hacia las que 
muchos de esos grupos fueron convergiendo progresivamente (aunque el más tradicional Stahlhelm, fundado en 1918, se mantiene activo hasta la llegada de Hitler al poder). Un nuevo fracaso (golpe de Múnich de noviembre de 1923, encabezado ya por Hitler) tampoco condujo al desmantelamiento de las redes paramilitares, aunque sí dio paso a cambios en el perfil del fenómeno: la transformación de las SA en un instrumento de propaganda politica mediante la violencia y la aparición mimética y defensiva de grupos paramilitares comunistas o incluso defensores de la República. Desde 1929/30 las SA desencadenan una "guerra civil latente", una "lucha por el control de las calles y el espacio público", en la que son combatidos sobre todo por los comunistas. El autor analiza con plasticidad la composición, la organización, el espíritu y las actividades de las SA en ese periodo, para terminar recordando que Hitler liquidó ese paramilitarismo ("noche de los cuchillos largos") cuando, dueño ya del poder total, pudo disponer de un sistema policial efectivo y crear un ejército poderoso.

La catedrática de Historia Stefanie Schüller-Springorum, directora del Centro de Investigaciones sobre Antisemitismo de la Universidad Técnica de Berlin, escribe "De las palabras a los hechos. Antisemitismo en la República de Weimar" (pp. 197-215). Este fenómeno se presenta como una manifestación peculiar del llamado "antisemitismo moderno", enraizado en las transformaciones sociales y económicas del siglo XIX como una forma particular de rechazo a la modernidad (laica, liberal, democrática) y dotado de un especial contenido político allí donde, como ocurría en Alemania, la exclusión jurídica de los judíos formaba parte de la memoria colectiva inmediata (solo en $1869 / 71$ se había suprimido formalmente). Durante todo este tiempo la segregación social se había mantenido, la "cuestión judia" seguía viva, el antisemitismo era socialmente aceptado. La Gran Guerra, con su impulso al nacionalismo, cortó de raíz los procesos de integración de un colectivo cuyo perfil transnacional le hacía sospechoso ante todos, pero que en Alemania concentraba además el resentimiento por la derrota; y generó también un movimiento migratorio de judios desde el este de Europa que estimuló las pasiones antisemitas.

La autora ofrece ilustrativos ejemplos de aislamiento, de rechazo y de violencia que no se limitan a los primeros años críticos. La exclusión social y las campañas y acciones antisemitas se enquistan durante el periodo central de la República y arraigan en el debate político de ámbito local, con el apoyo o la tolerancia de diversos grupos sociales y politicos y de la justicia. La escalada iniciada en 1930 (boicot comercial y laboral, violencia sistemática) se encuentra con un terreno abonado, sin que los judios contaran con otro respaldo que el implícito en la defensa del Estado democrático de Derecho por parte del partido socialista y del Zentrum católico. Dar publicidad a las informaciones, los análisis y las denuncias parecía reforzar la dinámica publicitaria antisemita; el sionismo y la lucha antifascista operaron como estrategias alternativas. Pero, finalmente, el acceso de Hitler al poder ofreció la posibilidad de convertir la difusa y muy difundida ideología antisemita en una práctica política sistemática y progresiva que apenas encontró resistencia.

Entre estas aportaciones de Ángel Alcalde y Stefanie Schüller-Springorum son apreciables afinidades temáticas y metodológicas. Lo mismo ocurre cuando se consideran los dos trabajos que componen la tercera parte del libro, escritos por Leonardo Álvarez, profesor de Derecho constitucional en la Universidad de Oviedo, y Leticia Vita, profesora de Teoría del Estado en la Universidad de Buenos Aires. 
En "Los poderes excepcionales del Presidente del Reich. El art. 48.2 de la Constitución de Weimar en el debate académico" (pp. 219-249) Leonardo Álvarez empieza por identificar la utilización abusiva de los poderes atribuidos por el precepto como una de las diversas causas a las que se suele atribuir el "naufragio" de la Constitución y de la República de Weimar. La investigación expone tanto los límites y controles previstos por el texto constitucional (situación habilitante y órgano habilitado, naturaleza y limites materiales de las medidas admisibles, temporalidad de las mismas, controles parlamentario y judicial) como el modo en que la práctica alteró el significado y dilató la operatividad de tales poderes. Pero procede, en particular, a analizar la doctrina que, comentando el precepto y su aplicación práctica, asumió las desviaciones como una práctica constitucional legítima.

Hay que recordar, en cualquier caso, que el art. 48.2 de la Constitución se invoca fundamentalmente en dos periodos distintos. Desde los primeros años de la República, el precepto, expresamente referido a la seguridad pública y el orden público, sirvió también para adoptar medidas orientadas a superar la crisis económica y financiera. En los años finales, cuando el uso de los poderes extraordinarios del Presidente y la parálisis del Reichstag se retroalimentan reciprocamente, el art. 48.2 se convierte en el eje sobre el que pivota la disyuntiva, irresuelta por la Constitución, entre plebiscitarismo identitario y régimen representativo parlamentario. Se entiende entonces que el Presidente puede sustituir con carácter general el ejercicio de las funciones atribuidas a otros órganos constitucionales, en particular al Reichstag, y eludir el control parlamentario de tales decretos de excepción ejerciendo su poder de disolución del Reichstag. Schmitt justifica además que estos decretos de excepción, cuya equiparación con la ley siempre se había admitido, puedan también apartarse de la "ley constitucional", diferente de la Constitución en su (para él) verdadero significado material.

Leonardo Álvarez subraya que, en efecto, desde la ponencia de Carl Schmitt en el Congreso de la Asociación de profesores alemanes de Derecho político de 1924, el art. 48.2 de la Constitución centra los debates acerca del concepto mismo de Constitución, como texto positivo que delimita las posibilidades de acción legítima o como decisión existencial que condiciona y en caso necesario desborda la interpretación del texto. Pero concluye su análisis sosteniendo que ambas opciones metodológicas abocaron a resultados prácticos similares (con matices, por ejemplo, al valorar la extensión de los poderes presidenciales en las materias objeto de reservas de ley), fuera interpretando de forma extensiva de las facultades otorgadas por el precepto o invocando poderes implícitos en la estructura material de la Constitución.

Al exponer la delimitación y el desbordamiento del ámbito de aplicación del art. 48.2 de la Constitución alude Leonardo Álvarez al mecanismo de ejecución federal previsto en el párrafo primero del mismo artículo, que quedó subsumido en el segundo por la resolución judicial del conflicto planteado contra la intervención del Reich en Prusia de 20 de julio de 1932. Precisamente este es el tema específico de la aportación de Leticia Vita, "El conflicto de Prusia contra el Reich” (pp. 251-274).

La autora menciona los datos que explican el conflicto (importancia relativa de Prusia en el Reich, diferente orientación de los respectivos gobiernos, interinidad del prusiano y apoyo exclusivamente presidencial para el federal de 
von Papen), describe los hechos desencadenantes ("el domingo sangriento de Altona") y expone la resolución del 20 de julio de 1932, el célebre Preußenschlag, que pretendió ampararse simultáneamente en los apartados 1 y 2 del art. 48 de la Constitución. También describe los términos en los que se planteó el debate ante el órgano jurisdiccional que conoció de la impugnación de tal decreto por parte de diversos sujetos legitimados, la competencia y composición del mismo y su resolución final, la sentencia de 25 de octubre que, pese a alguna concesión a la garantía del federalismo, entendió que el decreto estaba amparado en lo fundamental por el art. 48.2 de la Constitución (no por el párrafo primero).

Pero la atención de Leticia Vita se centra en los debates doctrinales, que en buena medida se articularon como disputa en sede judicial; porque, en efecto, entre los abogados de las partes se encontraban iuspublicistas tan notorios como, de un lado y entre otros, Gerhardt Anschütz, Friedrich Giese, Hans Nawiasky, Hans Peters y Hermann Heller; del otro, Carl Schmitt, Erwin Jacobi, Carl Bilfinger... Y destaca las argumentaciones desarrolladas ante el tribunal por Heller y Schmitt (perfilando también sus rasgos peculiares como oradores forenses), junto con las aportadas por Hans Kelsen en su inmediato comentario a la sentencia.

Para Heller, las medidas no estaban justificadas, pues las tensiones a las que pretendian responder no debían considerarse excepcionales, sino connaturales a la democracia pluralista. Pero ni siquiera admitiendo sus fines explícitos podían considerarse idóneas, necesarias y proporcionadas para alcanzarlos: constituían un "exceso de poder discrecional". Desvelaba así su verdadera finalidad politica y llamaba a los jueces a defender los principios constitucionales de la democracia social. Schmitt apela a la continencia judicial a la hora de afrontar cuestiones politicas, manipula los conceptos de autonomía y representación para discutir la posición procesal del Gobierno destituido en Prusia, que identifica como simple expresión de intereses de partido, y defiende la posición del Presidente del Reich como garante y protector de la Constitución y del Reich. Kelsen invoca, frente a la intervención del Reich, el federalismo y la democracia, que habrian quedado anulados en Prusia; y reprocha a la sentencia que no valorara la concurrencia de la situación de hecho que habilitaba para adoptar las medidas del art. 48.2 de la Constitución, además de otras inconsistencias. Pero, a su juicio, apenas cabía esperar algo mejor de la interpretación judicial de la Constitución cuando era esta misma la que no habia previsto una adecuada garantía jurisdiccional del régimen federal.

De este modo, ante un problema real y decisivo, Leticia Vita pone de manifiesto las implicaciones prácticas de los debates doctrinales sobre la jurisdicción constitucional y la defensa de la Constitución (Kelsen/Schmitt), y deja también en evidencia las correlaciones entre las formulaciones académicas y las posiciones politicas (Heller, Schmitt). Ella y Leonardo Álvarez, con un acercamiento directo a la doctrina determinante del periodo, permiten comprender la distancia exacta entre lo que se esperaba entonces de la Constitución y su rendimiento efectivo.

Enviado el (Submission Date): 01/05/2021

Aceptado el (Acceptance Date): 4/05/2021 